\title{
The evolution of fungal epiphytes
}

\author{
Hongsanan $\mathrm{S}^{1,2,3}$, Sánchez-Ramírez $\mathrm{S}^{4,5}$, Crous $\mathrm{PW}^{6}$, Ariyawansa $\mathrm{HA}^{7}$, Zhao \\ $\mathrm{RL}^{8}$, Hyde KD ${ }^{1,2,3^{*}}$
}

${ }^{1}$ Key Laboratory for Plant Diversity and Biogeography of East Asia, Kunming Institute of Botany, Chinese Academy of Sciences, Kunming 650201, Yunnan, People's Republic of China

${ }^{2}$ World Agroforestry Centre, East and Central Asia, Kunming 650201, Yunnan, People's Republic of China

${ }^{3}$ Center of Excellence in Fungal Research, Chiang Rai 57100, Thailand

${ }^{4}$ Department of Natural History, Royal Ontario Museum, Toronto, ON M5S 2C6, Canada

${ }^{5}$ Department of Ecology and Evolutionary Biology, University of Toronto, Toronto, ON M5S 3B2, Canada

${ }^{6}$ CBS-KNAW Fungal Biodiversity Centre, P.O. Box 85167, 3508 AD, Utrecht, The Netherlands

${ }^{7}$ Guizhou Key Laboratory of Agricultural Biotechnology, Guizhou Academy of Agricultural Sciences, Xiaohe District, Guiyang City, Guizhou Province 550006, People's Republic of China

${ }^{8}$ State Key Laboratory of Mycology, Institute of Microbiology, Chinese Academy of Sciences, Beijing 100101, People's Republic of China

Hongsanan S, Sánchez-Ramírez S, Crous PW, Ariyawansa HA, Zhao RL, Hyde KD. 2016 - The evolution of fungal epiphytes. Mycosphere 7 (11), 1690-1712, Doi 10.5943/mycosphere/7/11/6

\begin{abstract}
Fungal epiphytes are a polyphyletic group found on the surface of plants, particularly on leaves, with a worldwide distribution. They belong in the phylum Ascomycota, which contains the largest known number of fungal genera. There has been little research dating the origins of the common ancestors of fungal epiphytes. This study uses a molecular clock to provide a rough time frame for the origins of fungal epiphytes in the orders Asterinales, Capnodiales, Meliolales, Microthyriales and Zeloasperisporiales. LSU, SSU, RPB1 and RPB2 sequence data from representative strains of the major classes of Ascomycota are used to represent internal calibration points in the phylogenetic tree, to estimate divergence times of fungal epiphyte lineages. The estimated date crowns of fungal epiphytes included in the orders Asterinales, Capnodiales, Meliolales occur in the middle or the end of Jurassic, with Meliolales and Zeloasperisporiales occurring in the Cretaceous. Foliar epiphytes placed in totally unrelated classes evolved as early as the Permian (298.9 to 252.17 Mya) based on sequence data from representative foliar epiphytes and fossil calibrations. The evolution of the most closely related groups of fungi and foliar epiphytes occurred during the Triassic to Jurassic. Phylogenetic relationships, evolution of morphological characters and nutritional mode of foliar epiphytes are discussed.
\end{abstract}

Key words - BEAST - Fossil fungi - MrBayes - Phylogeny - Sooty molds - Taxonomy

\section{Introduction}

Fungal epiphytes are a polyphyletic group with a worldwide distribution (Schoch et al. 2009, Wu et al. 2011, Hyde et al. 2013, Hongsanan et al. 2014a, 2015a, b, c, Li et al. 2016). They are defined as specialized nutritional guilds found on the surface of living plant parts, particularly on leaves; including saprobes, plant parasites, fungal parasites and lichens (Gilbert \& Reynolds 2002, 2005). Many fungal epiphytes are obligate parasites (Wu et al. 2011, Hongsanan et al. 
2015a), which can damage the host plants by penetrating host cells for the uptake of nutrients (Ariyawansa et al. 2015, Hongsanan et al. 2014a, 2015a, b). Some species are saprobes and cause marketability problems, due to the black hyphae coating the surface of plants, especially economic fruits. Furthermore, they reduce photosynthetic ability of plants through the hyphal cover; they can also cause chlorosis under the hyphae and can cause plant-stunting disease and lower yield (Chomnunti et al. 2014, Hongsanan et al. 2015c, d). Some fungal epiphytes cause sooty blotch and flyspeck disease on surface of apple or other host plants such as mango and pears (Ismail et al. 2016).

Molecular clock methods have been used in several studies to date the origin and subsequent evolution of lineages in many groups of micro- and macrofungi (Berbee \& Taylor 1993, 2007, 2010, Heckman et al 2001, Sanderson 2003, Taylor \& Berbee 2006, Vijaykrishna et al. 2006, Beimforde et al. 2014, Zhao et al. 2016). The first application of the molecular clock to fungal groups was provided by Simon et al. (1993). Fossil evidence is essential for dating (Benton et al. 2009, Hedman 2010, Inoue et al. 2010, Magallon 2010, Pyron 2010, Wilkinson et al. 2011, Lukoschek et al. 2012, Sauquet et al. 2012). Fossil evidence is used as the minimum age, which results in calibration points in the phylogenetic tree (Marshall 2008, Forest 2009, Parham et al. 2012, Sauquet et al. 2012, Beimforde et al. 2014). Despite several recent studies dating fungi, molecular clock dating and studies on the evolution of fungal epiphyte lineages has been poorly studied.

In this study, we focus on the evolution of fungal epiphytes using molecular clock dating. The fungal epiphytes are black mildews, black dots, and sooty moulds, mostly on plant leaves and belong in the orders Asterinales, Capnodiales, Microthyriales and Zeloasperisporiales of Dothideomycetes (Hyde et al. 2013, Chomnunti et al. 2011, 2014, Hongsanan et al. 2014a, b, 2015a, b, c, d), and Meliolales of Sordariomycetes (Kirk et al. 2001, Justavino et al. 2015, Hongsanan et al. 2015a, Maharachchikumbura et al. 2015, 2016). We used multi-calibrations distributed across the Ascomycota as used in Pérez-Ortega et al. (2016), and in addition we used fossil evidence for fungal epiphytes from previous studies to strengthen the calibration.

\section{Fossil studies on fungal epiphytes}

\section{Asterinales}

Species in Asterinales are pathogenic biotrophs, appearing as black colonies on the surface of plants, particularly on leaves, and are common in tropical and subtropical regions and have a worldwide distribution )Hyde et al. 2013, Hongsanan et al. 2014a(. Although Asterinales appear to be similar to sooty moulds when observed with the unaided eye, they produce black, web-like colonies on leaves (Fig. 1), and cause minor damage to host plants by penetrating host cells for the uptake of nutrients. Sooty moulds however, feed on honeydew excreted from insects )Hughes 1976, Reynolds 1998, Chomnunti et al. 2012, 2014(. Members of Asterinales are host-specific biotrophs (Hongsanan et al. 2014a). A recent monograph of Asterinales was provided by Hongsanan et al. (2014a). The order presently comprises three families based on phylogenetic inferences: Asterinaceae, Melaspileaceae, and Parmulariaceae (Guatimosim et al. 2015).

Engelhardt \& Kinkelin (1908) studied fossils of leaves of Ilex (Aquifoliaceae), Sequoia (Cupressaceae), Sapindus (Sapindaceae), and Chrysobalanus (Chrysobalanaceae) and dated them to the Pliocene. They found taxa typical of Asterina on the fossil specimens. Thus, they concluded that the genus Asterina existed during the Pliocene (5.333 to 2.58 Mya). Dilcher (1965) found Asterina species on angiosperm leaves collected from lower Eocene deposits (56 to 33.9 Mya) in western Tennessee (USA), and introduced two new species, Asterina nodosaria Dilcher and A. eocenica Dilcher from Sapindus and Chrysobalanus, respectively. Dilcher (1965) also compared these asterina-like taxa from fossil specimens with modern specimens, and concluded that the genus Asterina originated at least as early as the Eocene. There are very few studies on both fossil and modern specimens of Asterinales. Consequently, they are poorly studied from an evolutionary point of view. 



Fig. 1 - Asterinales on the surface of various hosts. a Asterina species (Asterinaceae) on Acacia sp. b Asterina species (Asterinaceae) on unidentified leaves. c Asterina species (Asterinaceae) on Tinospora sp. d Aldona species (Parmulariaceae) on Pterocarpus draco. e Asterina species (Asterinaceae) on Pterocarpus sp.

Families of sooty moulds

Sooty moulds are saprobes, which indirectly damage host plants by reducing photosynthesis. Sooty moulds species normally feed on carbon sources, such as sugars (honeydew) excreted from sap-feeding insects; aphids, and whiteflies )Hughes 1976, Reynolds 1998, Chomnunti et al. 2011, 2012, 2014(. These taxa appear as a black mold covering the host surface with dark hyphae (Fig. 2); they can affect many economic crops, e.g. Capnodium citri on Citrus spp. (Reynolds 1999) and Sorias spp. on mangoes. Species that occur on the surface of plants especially on fruits can reduce their quality, have important implications for import and export of fruits and reduce the sale of fruits in markets )Chomnunti et al. 2014(. Sooty mould species are polyphyletic and comprise seven families based on morphology and phylogeny (Reynolds 1998, Winka et al. 1998, Hughes \& Seifert 2012, Hyde et al. 2013, Chomnunti et al. 2001, 2012, 2014); Antennulariellaceae, Capnodiaceae, Euantennariaceae, Metacapnodiaceae (Dothideomycetes) and Chaetothyriaceae, Coccodiniaceae, and Trichomeriaceae (Eurotiomycetes). Although they belong in two classes, they live differently and are unrelated. The ascomata of Chaetothyriales are surrounded by a pellicle of superficial mycelium, and are often multilocular. However, some taxa in Capnodiales have also been isolated as rock-dwelling fungi and were noted that some of these taxa perhaps evolved from capnodiaceous species (Ruibal et al. 2009, Selbmann et al. 2014). These questions need to be clarified by using the life cycle of all the families of sooty moulds. In this study, we focus on the foliar epiphytes of Dothideomycetes and Sordariomycetes, and Chaetothyriales will be studied in the future. 
The oldest known fossils of sooty moulds belong to the Metacapnodiaceae (Capnodiales) and were found in France in Early Cretaceous Charentes amber (145 \pm 4 to $66 \mathrm{Mya})$ and were dated to be at least 100 Mya (Néraudeau et al. 2002, Perrichot et al. 2010, Beimforde et al. 2014, Schmidt et al. 2014, Pérez-Ortega et al. 2016). Some of the taxa isolated as rock-dwelling fungi probably evolved from capnodiaceous sooty moulds (Ruibal et al. 2009, Selbmann et al. 2014). This can be clarified by molecular data when sufficient informative data become available.

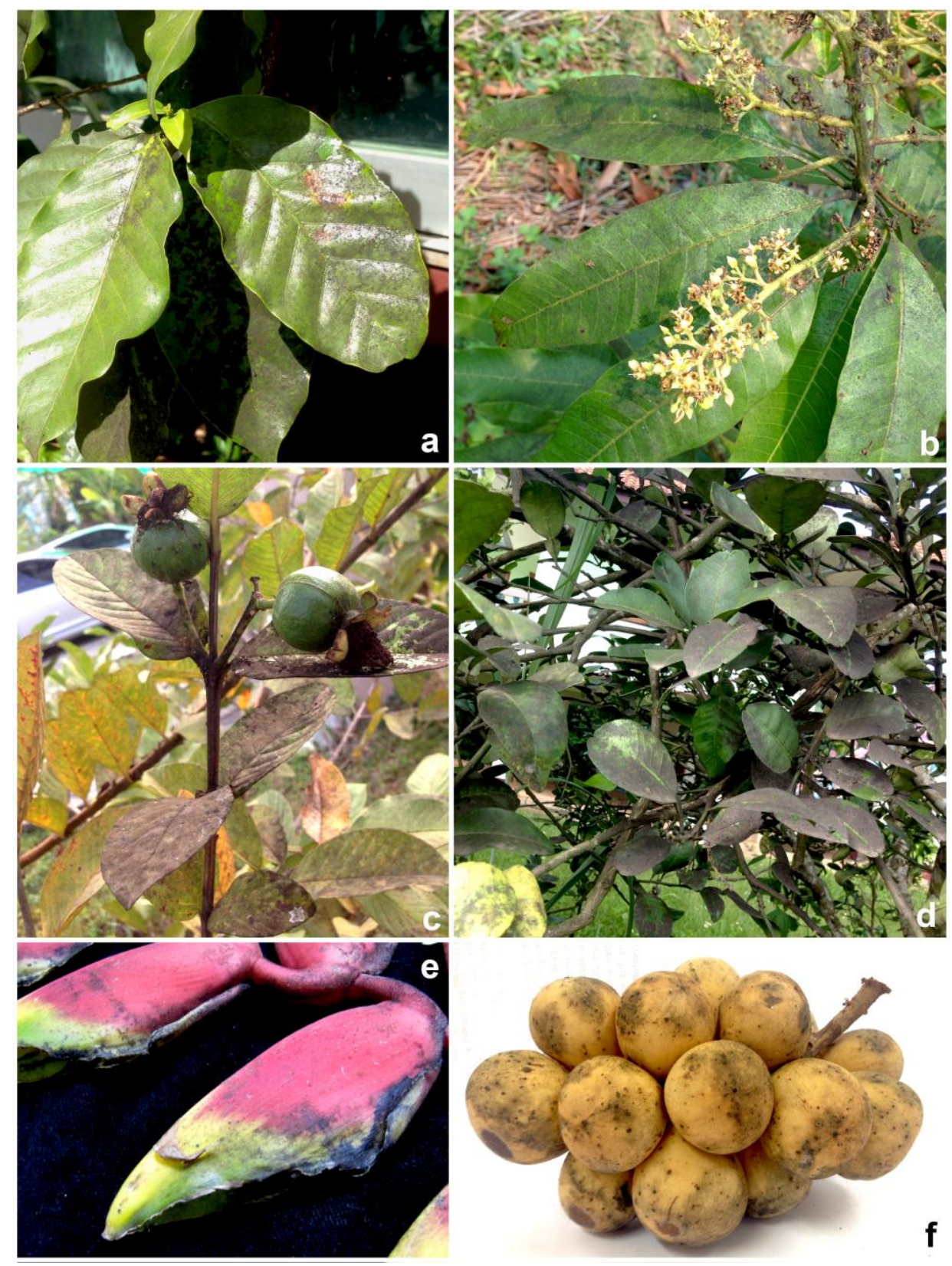

Fig. 2 - Sooty moulds on various hosts. a Coffea arabica. b Mangifera indica. c Psidium guajava. d Citrus maxima. e Heliconia sp. f Lansium domesticum.

\section{Meliolales}

The order Meliolales accommodates species of biotrophic epiphytes (Hansford 1961, Hongsanan et al. 2015a), which may cause leaves to become stunted and pale in colour. Some species do not produce pathogenic effects, but reduce photosynthetic efficiency and aesthetic beauty of the host plant (Fig. 3) (Thomas et al. 2013, Hongsanan et al. 2015a). Hongsanan et al. (2015a) monographed the order based on morphology and phylogeny and concluded that Meliolales presently contains Armatellaceae and Meliolaceae. The family Armatellaceae comprises a single genus, while Meliolaceae contains seven genera (Hongsanan et al. 2015a). Species are believed to 
be host-specific, however, Hongsanan et al. (2015a) have shown that Meliola thailandicum can occur on at least two host families. Phylogenetic analysis indicates that Meliolales is a subclass of Sordariomycetes, Meliolomycetidae (Kirk et al. 2001, Justavino et al. 2015, Hongsanan et al. 2015a, Maharachchikumbura et al. 2015, 2016), and this was confirmed by Maharachchikumbura et al. (2015). Meliolales species are biotrophic and cannot be cultured, thus there are few sequences for the species in GenBank.

There is little fossil evidence for Meliolales (Dilcher 1965). Meliola ellis Roum. was introduced as a species from fossils in northern England dated to the Holocene (0.0117 to 0 Mya) (Roumeguère 1880). Köck (1939) described meliola-like taxa from Eocene fossils (56 to 33.9 Mya) on Taxus L. (Taxaceae), Sapindus L. (Sapindoideae), Chrysobalanus L. (Chrysobalanaceae) and unidentified leaves. Dilcher (1965) established two new species, Meliola spinksii Dilcher and $M$. anfractus Dilcher, based on the comparison between fossil specimens discovered from the Eocene of Tennessee deposits.

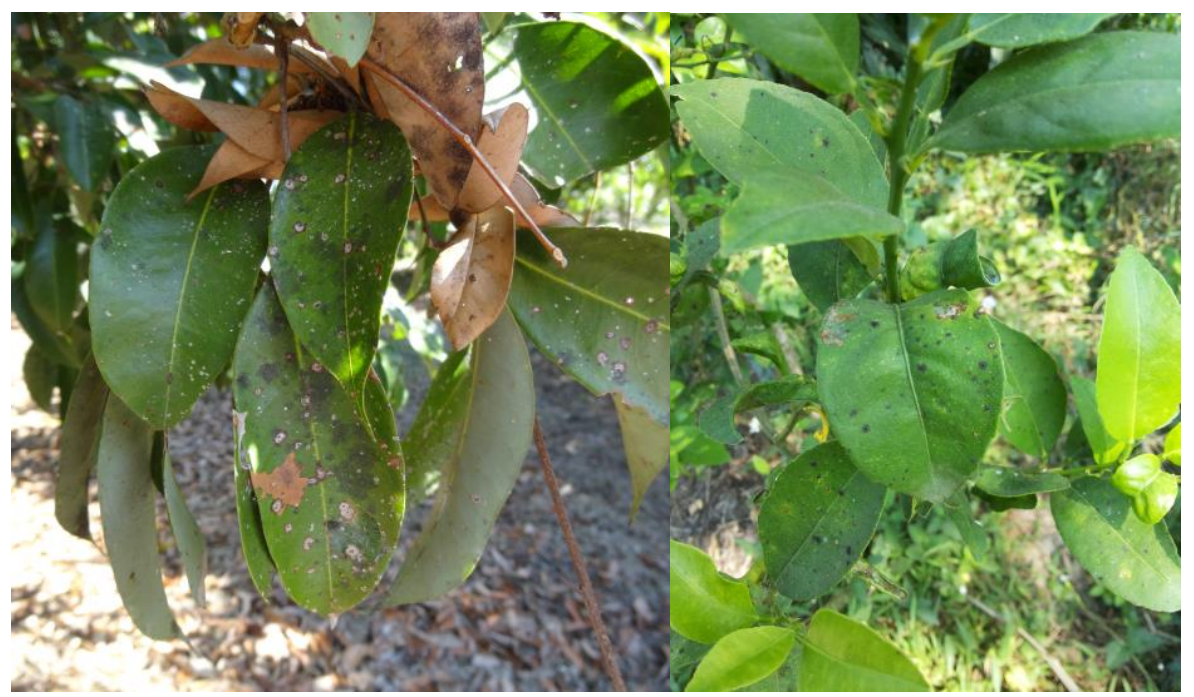

Fig. 3 - Meliolales on various hosts. a Litchi chinensis. b Citrus sp.

Microthyriales

Species in Microthyriales are fungal epiphytes including biotrophs and saprotrophs, usually found on leaves or fruits (Wu et al. 2011, Hongsanan et al. 2014b). They indirectly affect host plants by penetrating and extracting nutrients, but do not cause much damage. Species of Microthyriales appear as on leaves as small, circular, flattened, black dots, with a prominent central ostiole, and are poorly developed at the base. They are easily removed from the surface of the host. Asci are bitunicate and ascospores are 1-septate, some with appendages (Arnaud 1918, Luttrell 1973, von Arx \& Müller 1975, Barr 1987, Kirk et al. 2008, Wu et al. 2011, Hyde et al. 2013). The appearance of Microthyriales species on fruits can reduce marketability. Wu et al. )2011( recognized seven genera of Microthyriaceae, while a further four genera were added by Hyde et al. )2013(. A further two new genera were added by Hongsanan et al. )2014b( and Ariyawansa et al. (2015) with evidence from morphology and phylogeny. A few species in Microthyriales have been sequenced and higher level placement of the order has partially been resolved. However, there are many species in Microthyriales that have not been sequenced. Frantz (1959) reported microthyriaceous species on Sapindus, Pityophyllum (Gnetopsida) and numerous unidentified leaves from the fossil Tertiary (66 to 2.58 Mya). Fossilized Microthyrium on Buxus protojaponica was found in Japan in the Miocene (23.03 to 5.332 Mya) by Doi and Uemura (1985). Szafer (1961) and Lancucka-Srodoniowa (1966) described Microthyriales on Buxus sp. from the fossil Miocene of Europe. Godwin and Andrew (1951) reported Microthyrium on a Buxus fossilized leaf in England from the Plaeistocene (2.58 to $0.0117 \mathrm{Mya}$ ). Some fossil specimens were described from the Plaeistocene under the form-name Microthyriacites or Phragmothyrites (Givulescu 1971, Cooksoon 1947, Selkirk 1975). Microthyrialean taxa are common in the Eocene (56 to 33.9 Mya) 
or younger than Eocene (Germeraad 1979, Doi \& Uemura 1985). However, the oldest fossil of the order Microthyriales was recorded in Colorado from the upper Cretaceous (145 \pm 4 to 66 Mya) (Eriksson 1978).

Zeloasperisporiales

The order was established to accommodate a single family Zeloasperisporiaceae by Hongsanan et al. (2015b). Species of Zeloasperisporiaceae appear as small black dots on the surface of the host, often similar to species in Microthyriales. The generic type of this family is Zeloasperisporium which was introduced based on asexual characters. The life cycle of Zeloasperisporium species are remarkable as they have been isolated from the air and leaves and may obtain nutrients from plant cells using appressorium-like, inflated hyphopodia, which are slightly warted to lobed at the apex (Castañeda et al. 1996). However, Crous et al. (2007) recognized this structure as conidiogenous cells of a synanamorph forming a second conidial type. The sexual and asexual morphs of Zeloasperisporiaceae species were clarified and discussed by Hongsanan et al. (2015b). There is no fossil evidence for this order. Some taxa were discovered on amber fossil and resemble to Zeloasperisporiales species such as Callimothallus pertusus Dilcher which has flattened stromata on upper epidermis of leaf of Sapindus sp. (Sapindaceae), but associated with hyphae of Shortensis memorabilis Dilcher. Thus, we cannot be sure that they belong in Zeloasperisporiales due to the poor condition of specimens.

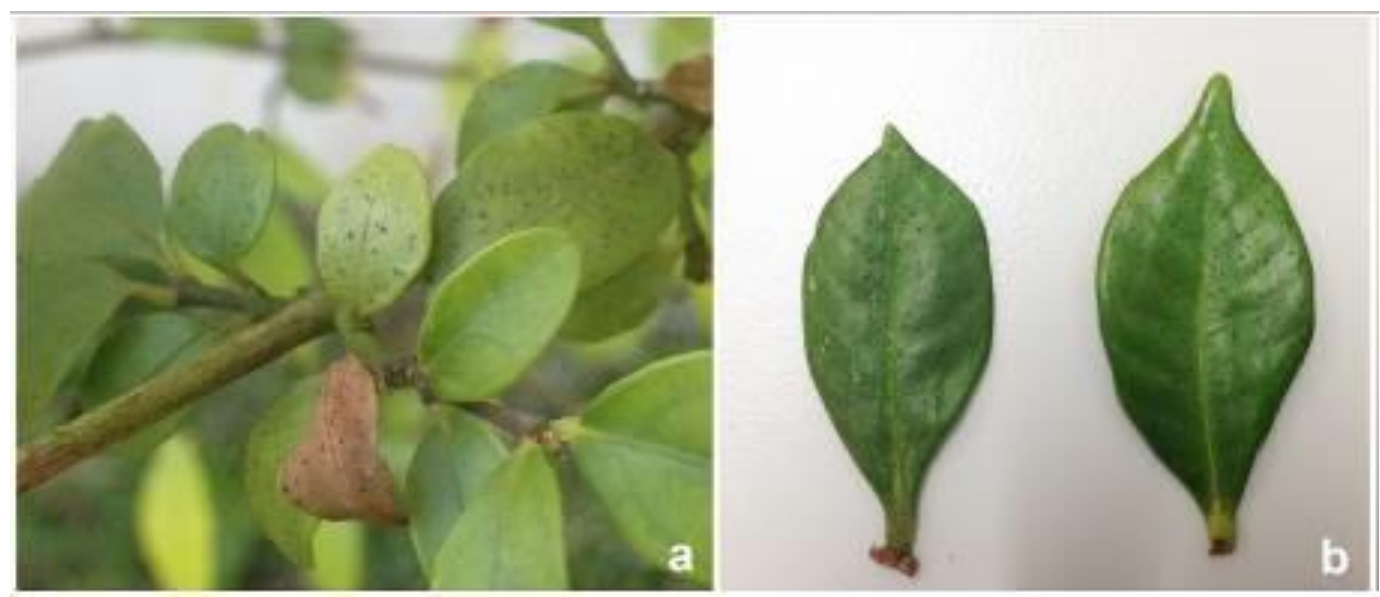

Fig. 4 - Zeloasperisporiales species on living leaves. a On Wrightia religiosa. b On unidentified leaves

\section{Materials \& Methods}

\section{Phylogenetic analyses}

LSU, SSU, and RPB2 sequence data from the representative major orders in Dothideomycetes and Sordariomycetes were obtained from GenBank (Table. 1). The molecular clock tree was divided into two trees, which are fungal epiphytes in Sordariomycetes and Dothideomycetes.

The representative strains from Dothideomycetes were downloaded from GenBank. The representative strains from Leotiomycetes were selected as outgroup. The data set was aligned by using MAFFT (Katoh et al. 2009), checked and aligned manually using Bioedit (Hall 1999). The jModeltest was used to perform to select the best-fit models of nucleotide substitution for each gene. Initial phylogenetic tree was performed by using MCMC sampling in MrBayes v3.1.2 (Huelsenbeck \& Ronquist 2001, Zhaxybayeva \& Gogarten 2002), following Cai et al. (2006, 2008). The analysis used 4 nchains, and run for 10,000,000 generations. Trees were sampled every $1000^{\text {th }}$ generation which produces 10,000 trees. The first 2,000 trees are known as burin-in phase, and were discarded, and the remaining 8,000 trees were used to calculate the posterior probabilities. 
The representative strains from Sordariomycetes were downloaded from GenBank. The representative strains from Lecanoromycetes were selected as outgroup taxon. The data set was aligned using the same methods with the Dothideomycetes tree. The jModeltest was used in combination with AIC to estimate the best nucleotide substitution model the recommended models in the dataset of Sordariomycetes were GTR $+\mathrm{I}+\mathrm{G}$ for LSU and RPB2, SYM+I+G for SSU. An initial phylogenetic tree was prepared using the same methods mentioned above.

\section{Fossil calibrations}

Divergence time estimation analyses were performed using the fossil calibrations as in Beimforde et al. (2014) and Pérez-Ortega et al. (2016). The results from Pérez-Ortega et al. (2016) were used as secondary calibration in this study. Estimating divergence time of the common ancestor of fungal epiphytes in Dothideomycetes and Sordariomycetes was performed separately using BEAST for evolution analysis.

To estimate the molecular clock tree of fungal epiphytes in the Dothideomycetes, the Dothideomycetes crown group was calibrated using the result from Pérez-Ortega et al. (2016) as secondary calibration (normal distribution, mean $=290, \mathrm{SD}=30$, with providing $95 \%$ credibility interval of $339 \mathrm{Mya}$ ). Metacapnodiaceae was used as a minimum age of Capnodiales (normal distribution, mean $=100, \mathrm{SD}=150$, with providing 95\% credibility interval of 346 Mya.). Microthyrium microscopicum and M. buxicola are typical of the oldest fossil of Microthyrium, thus used as the oldest fossil to be the minimum age of the common ancestor of Microthyriales (gamma distribution, shape $=1$, scale $=50$, offset $=65$, with providing $95 \%$ credibility interval of 215 Mya.). The divergence times of the genus Calicium was estimated by Pérez-Ortega et al. (2016) and it was used as the secondary calibration in this study (Gamma, mean $=35, \mathrm{SD}=40$, with providing $95 \%$ credibility interval of $155 \mathrm{Mya}$ ).

To estimate the molecular clock tree of fungal epiphytes in the Sordariomycetes, the rootHeight parameter was calibrated from the split of Leotiomycetes and Sordariomycetes (gamma distribution, shape $=1$, scale $=50$, offset $=300)$. The Sordariomycetes crown group was calibrated to be 256 Mya (202-306) by Pérez-Ortega et al. (2016), (normal distribution, mean $=250, \mathrm{SD}=50$, with providing 95\% credibility interval of 332 Mya). Dilcher (1965) studied the Meliola species on fossil Eocene, Meliola spinksii, and it is similar to modern specimens of M. thailandicum. However, setae with branches at the apex are present in $M$. thailandicum, while they are undetermined in fossil specimens. Based on the similarity of fossil specimens and modern specimens, we assumed that the genus Meliola had existed in the Eocene (gamma distribution, shape $=1$, scale $=50$, off set $=35$ ).

\section{Molecular clock analysis}

Molecular clock analyses were performed using BEAST 1.8.0. Aligned sequence data were partitioned separately for each LSU, SSU and RPB2 data set, and loaded to BEAUti 1.8.0. The data partitions were set with unlinked substitution, models and unlinked clock model and linked tree based on jModeltest results. Taxa sets were created for each interested groups and calibration of the common ancestor nodes, statistics associated with the most recent common ancestor (TMRCA). Substitution model was specified for each of data partitions (GTR+I+G for all genes used in Dothideomycetes, GTR+I+G for LSU and RPB2 and SYM+I+G for SSU used in Sordariomycetes). We used a lognormal distribution of rates for each gene estimated during the analyses with uncorrelated relaxed clock model (ucld). The tree prior was shared by all tree models, and consisted in a birth/death incomplete sampling tree prior was used to model the speciation of nodes in the topology. The analyses were performed for 50 million generations for both Dothideomycetes and Sordariomycetes, and sampling parameters every 1000 generations. Tracer v.1.6 was used to check the effective sample sizes, acceptable values were higher than 200 . The first 50,000 trees representing the burn-in phase were discarded. The remaining trees were combined in LogCombiner 1.8.0. A maximum clade creditability (MCC) tree was given by summarized data, tree was estimated in TreeAnnotator 1.8.0. The tree was then viewed in FigTree (Rambaut 2006). 
Table 1 Taxa used in the phylogenetic analysis and GenBank accession numbers (LSU, SSU, RPB2) and species voucher/culture numbers.

\begin{tabular}{|c|c|c|c|c|}
\hline \multirow{2}{*}{ Species } & \multirow{2}{*}{ Voucher/culture } & \multicolumn{3}{|c|}{ Accession numbers } \\
\hline & & LSU & SSU & RPB2 \\
\hline Acrospermum compressum & M151 & EU940084 & EU940237 & EU940301 \\
\hline Acrospermum gramineum & M152 & EU940085 & EU940238 & EU940302 \\
\hline Alternariaster bidentis & CBS 134021 & KC609341 & & КС609347 \\
\hline Amphibambusa bambusicola & MFLUCC 11-0617 & KP744474 & & \\
\hline Annulusmagnus triseptatus & CBS:128831 & JQ429242 & & JQ429258.1 \\
\hline Antennariella placitae & CBS 124785 & GQ303299 & & \\
\hline Ascovaginospora_stellipala & P5-13A & U85088 & U85087 & \\
\hline Asteridiella obesa & VIC:31239 & JX096809 & & \\
\hline Asterina fuchsiae & TH 590 & GU586216 & GU586210 & \\
\hline Asterina phenacis & TH 589 & GU586217 & GU586211 & \\
\hline Bambusicola massarinia & MFLUCC 11-0389 & JX442037 & JX442041 & KP761716 \\
\hline Bambusicola splendida & MFLUCC 11-0439 & JX442038 & JX442042 & \\
\hline Bombardia bombarda & AFTOL_ID_967 & DQ470970 & DQ471021 & DQ470923 \\
\hline Botryosphaeria agaves & MFLUCC $1 \overline{1}-0125$ & NG_042723 & JX646825 & \\
\hline Botryosphaeria dothidea & CBS 115476 & DQ3777852 & DQ677998 & DQ677944 \\
\hline Botryosphaeria tsugae & AFTOL-ID 1586 & DQ767655 & & DQ767644 \\
\hline Botryotinia fuckeliana & spat 03-11 & AY544651 & AY544695 & DQ247786 \\
\hline Calicium salicium & CBS: 100898 & & KF157982 & KF157998 \\
\hline Calicium viride & U. Soechting 7475 & AF356670 & & \\
\hline Camarosporium_quaternatum & CBS 483.95 & GU301806 & GU296141 & \\
\hline Capnodium coartatum & MFLUCC10-0069 & JN832614 & JN832599 & \\
\hline Capnodium salicinum & AFTOL-ID937 & DQ678050 & DQ677997 & \\
\hline Caryospora minima & - & EU196550 & EU196551 & \\
\hline Catabotrys deciduum & SMH3436 & AY346268 & & AY780158 \\
\hline Cephalotheca foveolata & UAMH11631 & KC408398 & & KC408404 \\
\hline Chaetomidium galaicum & CBS: 113678 & FJ666361 & & FJ666392 \\
\hline Chaetosphaerella fusca & GKML124N & FJ968967 & & \\
\hline Chaetosphaeria innumera & SMH 2748 & AY017375 & & \\
\hline Chromendothia citrina & AFTOL-ID 2121 & & DQ862046 & \\
\hline Conidiocarpus caucasicus & GUMH937 & KC833050 & KC833051 & \\
\hline Coniochaeta ligniaria & $\mathrm{C} 8$ & AY198388 & & \\
\hline Coniochaeta ostrea & AFTOL-ID 915 & DQ470959 & DQ471007 & DQ470909 \\
\hline Cordana abramovii & PE 0053-24a & KF833358 & & \\
\hline Cordana inaequalis & CBS 508.83 & HE672157 & & \\
\hline Coronophora gregaria & ANM1555 & & & FJ968938.1 \\
\hline Corynascella inaequalis & CBS 284.82 & & & HQ871839 \\
\hline Corynespora cassiicola & CBS 100822 & GU301808 & GU296144 & GU371742 \\
\hline Corynespora smithii & CABI 5649b & GU323201 & & GU371783 \\
\hline Cryptendoxyla hypophloia & WM10.89 & HQ014708 & & \\
\hline Cryptodiaporthe aesculi & AFTOL_ID_1238 & DQ836905 & DQ836899 & DQ836892 \\
\hline Cryptosphaerella elliptica & $\mathrm{SMH} 47 \overline{2} 2$ & FJ968974 & & FJ968944 \\
\hline Cucurbitaria berberidis & MFLUCC 11-0387 & КС506796 & KC506800 & \\
\hline Curvularia brachyspora & MFLU 14-0013 & KU746805 & KU746807 & KU746809 \\
\hline Cyphelium inquinans & Tibell 22283 (UPS) & AY453639 & U86695 & \\
\hline Cyphelium tigillare & Tibell 22343 (UPS) & AY453641 & AF241545 & \\
\hline Cyphellophora laciniata & AFTOL-ID 1033 & EF413619 & EF413618 & \\
\hline Cystocoleus ebeneus & L161 & EU048578 & EU048571 & \\
\hline Cytospora elaeagni & CFCC_89633 & KF765693 & & KU710956 \\
\hline Dermea acerina & CBS $1 \overline{6} 1.38$ & DQ247801 & DQ247809 & DQ247791 \\
\hline Diaporthe eres & AR3519 & AF362565 & & \\
\hline Diaporthe phaseolorum & NRRL_13736 & & & AY641036 \\
\hline Diatrype disciformis & AFTOL-ID 927 & DQ470964 & DQ471012 & DQ470915 \\
\hline Diatrype palmicola & MFLUCC 11-0020 & KP744482 & KP753950 & \\
\hline Didymella exigua & CBS 183.55 & JX681089 & EU754056 & GU371764 \\
\hline Didymosphaeria rubi-ulmifolii & MFLUCC 14-0023 & KJ436586 & KJ436588 & \\
\hline Dothiora cannabinae & AFTOL-ID 1359 & DQ470984 & DQ479933 & DQ470936 \\
\hline Echinosphaeria_canescens & SMH 4791 & AY436403 & & \\
\hline
\end{tabular}




\begin{tabular}{|c|c|c|c|c|}
\hline \multirow{2}{*}{ Species } & \multirow{2}{*}{ Voucher/culture } & \multicolumn{3}{|c|}{ Accession numbers } \\
\hline & & LSU & SSU & RPB2 \\
\hline Elsinoe centrolobi & CBS 222.50 & DQ678094 & DQ678041 & \\
\hline Elsinoe fawcettii & CPC 18535 & JN940382 & JN940559 & \\
\hline Elsinoe phaseoli & CBS 165.31 & DQ678095 & DQ678042 & KT216560 \\
\hline Elsinoe verbenae & СРC 18561 & JN940391 & JN940562 & \\
\hline Endomeliola dingleyae & PDD 98304 & GU138866 & & \\
\hline Endothia gyrosa & AFTOL-ID 1223 & DQ470972 & DQ471023 & DQ470926 \\
\hline Exserticlava vasiformis & TAMA 450 & AB753846 & & \\
\hline Extremus antarcticus & CCFEE 5312 & KF310020 & & KF310086 \\
\hline Gelasinospora tetrasperma & CBS 178.33 & DQ470980 & DQ471032 & DQ470932 \\
\hline Gnomonia gnomon & CBS 199.53 & AF408361 & DQ471019 & DQ470922 \\
\hline Gondwanamyces capensis & CMW997 & KM495391 & & \\
\hline Gondwanamyces proteae & CMW738 & KM495393 & & \\
\hline Helicascus nypae & BCC 36751 & GU479788 & GU479754 & GU479826 \\
\hline Helminthosphaeria hyphodermae & SMH4192 & KF765608 & & \\
\hline Hydropisphaera erubescens & ATCC 36093 & AY545726 & AY545722 & AY545731 \\
\hline Hypocrea americana & AFTO-ID 52 & AY544649 & AY544743 & \\
\hline Irenopsis walsurae & MFLU13-0621 & KT021648 & KT021648 & \\
\hline Irenopsis cornuta & VIC32058 & KC618642 & KC618657 & \\
\hline Irenopsis vincensii & VIC:31751 & JX133163 & & \\
\hline Jobellisia guangdongensis & GD14-4 & JN936990 & & \\
\hline Jobellisia luteola & SMH2753 & AY346286 & & \\
\hline Jugulospora rotula & ATCC 38359 & AY346287 & & AY780178 \\
\hline Julella avicenniae & BCC 20173 & GU371822 & GU371830 & GU371786 \\
\hline Karschia cezannei & Cezanne-Eichler 7453 & KP456153 & & \\
\hline Katumotoa bambusicola & KT $1517 \mathrm{a}$ & AB524595 & AB524454 & AB539095 \\
\hline Kellermania yuccigena & CBS 131727 & KF766356 & KF766272 & \\
\hline Kylindria peruamazonensis & CBS 838.91 & GU180638 & GU180609 & GU180656 \\
\hline Labrocarpon canariense & Ertz 16308 (BR) & KP456157 & & \\
\hline Lachnum virgineum & CBS:122031 & AY544646 & AY544688 & DQ470877 \\
\hline Lentithecium fluviatile & CBS 123090 & FJ795450 & FJ795492 & FJ795467 \\
\hline Leptosphaeria doliolum & MFLUCC: 151875 & KT454719 & KT454734 & \\
\hline Leptosphaerulina australis & CBS 317.83 & EU754166 & GU296160 & GU371790 \\
\hline Leptoxyphium cacuminum & MFLUCC10-0049 & JN832602 & JN832587 & \\
\hline Leptoxyphium cacuminum & MFLUCC10-0049 & JN832602 & JN832587 & \\
\hline Leucostoma niveum & AR3413 & AF362558 & & \\
\hline Lindra thalassiae & JK 5090A & DQ470947 & DQ470994 & DQ470897 \\
\hline Lophiotrema nucula & CBS 627.86 & GU301837 & GU296167 & FJ795463 \\
\hline Lophium mytilinum & AFTOL-ID 1609 & DQ678081 & DQ678030 & DQ677979 \\
\hline Lulworthia fucicola & ATCC 64288 & AY878965 & AY879007 & \\
\hline Magnaporthe salvinii & M21 & JF414862 & JF414887 & \\
\hline Manglicola guatemalensis & BCC20157 & FJ743450 & FJ743444 & \\
\hline Massarina eburnea & CBS 473.64 & GU301840 & GU296170 & GU371732 \\
\hline Mazzantia napelli & AR3498 & AF408368 & & \\
\hline Melanomma pulvis-pyrius & CBS 371.75 & GU301845 & FJ201989 & GU371798 \\
\hline Melanospora tiffanii & ATCC 15515 & FJ748915 & AY015619 & AY015637 \\
\hline Melanospora zamiae & ATCC 12340 & U17405 & AY046578 & AY046580 \\
\hline Melaspileopsis cf. diplasiospora & Ertz 16247 (BR) & KР456164 & & \\
\hline Meliola centellae & VIC:31244 & JQ734545 & & \\
\hline Meliola thailandicum & MFLU 15-0379 & KR868696 & & \\
\hline Microascus trigonosporus & AFTOL-ID 914 & DQ470958 & DQ471006 & DQ470908 \\
\hline Microsphaeropsis_olivacea & CBS 233.77 & GU237988 & & KT389643 \\
\hline Microthyrium buxicola & MFLUCC 15-0212 & КT306551 & KT306549 & \\
\hline Microthyrium microscopicum & CBS 115976 & GU301846 & GU296175 & GU371734 \\
\hline Mollisia cinerea & AFTOL-ID 76 & DQ470942 & DQ470990 & DQ470883 \\
\hline Monilinia fructicola & AFTOL-ID 169 & AY544670 & AY544714 & DQ470889 \\
\hline Murispora rubicunda & IFRD 2017 & FJ795507 & GU456308 & \\
\hline Muуocopron dipterocarpi & MFLUCC:14-1103 & KU726966 & KU726969 & \\
\hline Muyocopron lithocarpi & MFLUCC:14-1106 & KU726967 & KU726970 & \\
\hline Myriangium duriaei & CBS 260.36 & NG_027579 & AF242266 & KT216528 \\
\hline Myriangium duriaei & CBS 260.36 & NG_027579 & AF242266 & KT216528 \\
\hline
\end{tabular}




\begin{tabular}{|c|c|c|c|c|}
\hline \multirow{2}{*}{ Species } & \multirow{2}{*}{ Voucher/culture } & \multicolumn{3}{|c|}{ Accession numbers } \\
\hline & & LSU & SSU & RPB2 \\
\hline Myriangium hispanicum & CBS 247.33 & GU301854 & GU296180 & GU371744 \\
\hline Mytilinidion rhenanum & CBS 135.45 & FJ161175 & & \\
\hline Natarajania indica & GUFCC_5240 & HM171321 & & \\
\hline Natipusilla decorospora & AF236-1a & HM196369 & HM196376 & \\
\hline Natipusilla naponensis & AF217-1a & HM196371 & HM196378 & \\
\hline Nectria cinnabarina & CBS 114055 & & & DQ522456 \\
\hline Neocylindroseptoria pistaciae & CBS 471.69 & KF251656 & & KF252161 \\
\hline Nitschkia tetraspora & GKML148N & FJ968987 & & \\
\hline Ophioceras aquaticus & IFRDCC 3091 & JQ797433 & JQ797435 & \\
\hline Ophioceras commune & M91 & JX134687 & JX134661 & \\
\hline Ophiocordyceps sinensis & YN09 64 & JX968033 & JX968028 & JX968013 \\
\hline Ophiostoma piliferum & AFTOL-ID 910 & DQ470955 & DQ471003 & DQ470905 \\
\hline Parabambusicola thailandica & MFLUCC 11-0183 & KP744490 & KP753955 & \\
\hline Phaeodimeriella cissampeli & MFLU 16-0558 & KU746806 & KU746808 & KU746810 \\
\hline Phaeotrichum benjaminii & CBS 541.72 & AY004340 & AY016348 & GU357788 \\
\hline Phyllachora graminis & UME 31349 & & AF064051 & \\
\hline Phyllopsora sp. & AFTOL-ID 84 & KF157990 & KF157978 & \\
\hline Piedraia hortae & CBS 480.64 & GU214466 & & \\
\hline Plagiostoma euphorbiae & CBS 340.78 & AF408382 & DQ862055 & DQ368643 \\
\hline Platystomum crataegi & MFLUCC 14-0925 & KT026109 & KT026113 & \\
\hline Pleomassaria siparia & AFTOL-ID 1600 & DQ678078 & DQ678027 & DQ677976 \\
\hline Pleospora herbarum & IT 956 & KP334709 & KP334729 & KP334733 \\
\hline Pleurostoma ootheca & CMU 23858 & AY761079 & AY761074 & \\
\hline Pleurostomophora ochracea & CBS 131321 & JX073274 & JX073269 & \\
\hline Pleurostomophora richardsiae & CBS 270.33 & AY761080 & AY729812 & HQ878607 \\
\hline Podosordaria tulasnei & CBS 128.80 & KT281897 & & \\
\hline Preussia funiculata & CBS 659.74 & GU301864 & GU296187 & GU371799 \\
\hline Proxipyricularia zingiberis & HYZiM201-1-1-1 & KM484986 & & \\
\hline Pseudallescheria boydii & CBS 108.54 & EF151315 & & \\
\hline Pseudomassariosphaeria bromicola & IT-1333 & КT305994 & KT305996 & \\
\hline Pseudoproboscispora caudae-suis & A336-2D & AY094192 & & \\
\hline Pseudostrickeria muriformis & MFLUCC_13-0764 & КТ934254 & KT934258 & \\
\hline Pyricularia borealis & CBS $461 . \overline{65}$ & KM009150 & DQ341489.1 & \\
\hline Ramularia endophylla & CBS 113265 & KF251833 & EU167569 & KF252332 \\
\hline Rasutoria pseudotsugae & rapssd & EF114704 & EF114729 & \\
\hline Rasutoria tsugae ratstk & ratstk & EF114705 & EF114730 & \\
\hline Remispora maritima & ВBH28309 & HQ111012 & HQ111002 & HQ111041 \\
\hline Salsuginea_ramicola & KT 2597.1 & GU479800 & GU479767 & GU479833 \\
\hline Schizothyrium pomi & CBS 406.61 & EF134949 & EF134949 & \\
\hline Scortechinia acanthostroma & SMH1143 & FJ968988 & & FJ968948 \\
\hline Scortechiniellopsis leonensis & GKM1269 & FJ968993 & FJ968933 & \\
\hline Sillia ferruginea & AR_3440 & AR 3440 & & \\
\hline Slopeiomyces cylindrosporus & CBS̄ 609.75 & KM485040 & & KM485158 \\
\hline Sordaria fimicola & CBS 508.50 & AY681160 & & \\
\hline Stachybotrys chlorohalonata & UAMH6417 & AY489712 & AY489680 & \\
\hline Stictographa lentiginosa & Ertz $17447(\mathrm{BR})$ & KР456169 & & \\
\hline Sympoventuria capensis & CBS 120136 & KF156104 & KF156094 & \\
\hline Teratosphaeria fibrillosa & CBS 121707 & KF902075 & GU296199 & \\
\hline Tirisporella beccariana & BCC36737 & JQ655450 & JQ655454 & \\
\hline Trichodelitschia munkii & Kruys 201 (UPS) & DQ384096 & DQ384070 & \\
\hline Tubeufia chiangmaiensis & MFLUCC 11-0514 & KF301538 & KF301543 & \\
\hline Uwebraunia commune & NC1 32C1d & JQ622093 & & \\
\hline Valsa_ambiens & AR3516 & AF362564 & & \\
\hline Venturia inaequalis & CBS 476.61 & GU456336 & & \\
\hline Vialaea mangifia & MFLUCC 12-0808 & KF724975 & & \\
\hline Xenolophium_applanatum & CBS 123127 & GU456330 & GU456313 & GU456355 \\
\hline Xylaria hypoxylon & CBS 122620 & KM186301 & & \\
\hline Zeloasperisporium hyphopodioides & CBS 218.95 & EU035442 & & \\
\hline Zeloasperisporium siamemse & IFRDCC 2194 & JQ036228 & JQ036223 & \\
\hline Zeloasperisporium wrightiae & MFLUCC 15-0225 & KT387737 & KT387738 & \\
\hline
\end{tabular}




\section{Results}

\section{Phylogenetic analyses}

Phylogenetic analyses based on the LSU, SSU, and RPB2 sequence data of Dothideomycetes (Fig. 5) indicate that the species in Asterinales is placed within Dothideomycetes, and is distinct from other orders in the Dothideomycetes with high support (100\% ML). Asterinales is closely related to Botryosphaeriales, but this relationship is not well-supported. The order Capnodiales contains the largest family of sooty moulds which is Capnodiaceae. The order Capnodiales was represented by 12 strains in this study, consequently appear to be well-resolved within Dothideomycetes with high bootstrap support (100\% ML), and they formed a sister group to Myriangiales (83\% ML). The order Microthyriales comprises four representative strains, which clustered within the Dothideomycetes (100\% ML). Microthyriales is related to the clade of Natipusillales, which is fresh water fungi and fungal epiphytes Zeloasperisporiales, but its affinities to other orders are not well-resolved. Zeloasperisporiales species clustered with $100 \% \mathrm{ML}$ support and are closely related to Natipusillales (84\% ML), but as a distinct lineage from Microthyriales. However, Natipusillales and Zeloasperisporiales have very different morphology and habitats (Hongsanan et al. 2015b). The Sordariomycetes tree generated by maximum likelihood analysis from combined LSU, SSU, and RPB2 sequence data indicates that the Meliolales clade includes seven species from the family Meliolaceae, which are grouped and placed in Sordariomycetes with high support (100\% ML), which is congruent to the results of Hongsanan et al. (2015a) and Maharachchikumbura et al. $(2015,2016)$. Meliolales is closely related to the family Cephalothecaceae, which is placed as family incertae sedis in Sordariomycetes.

Divergence time estimates

In this study, the mean estimated dates are quite similar to previous studies (Beimforde et al. 2014; Pérez-Ortega et al. 2016). The MCC tree of Dothideomycetes provided by BEAST indicates that the divergence time estimated for Dothideomycetes and Lecanomycetes is 334 Mya (320-368), in the Carboniferous. The strains of Calicium, Cyphelium and Phycia grouped to represent the class Lecanoromycetes, which has an estimated date of 96 Mya (62-138) (Fig. 7). The calibrations for Lecanoromycetes in our analysis resulted in an estimated crown date for Dothideomycetes at 315 Mya (278-348), during the Carboniferous. The divergence times of fungal epiphytes within the class Dothideomycetes estimated in this study are shown in Table 2 . The order Asterinales was estimated with a crown date at 188 Mya (130-248), during the Jurassic. Furthermore, our analysis demonstrated that Asterinales shared the most common ancestor with Capnodiales, Dothideales, and Myriangiales at 270 Mya (226-315), in the Permian. The order Capnodiales diverged from Myriangiales at 205 Mya (166-248), in the terminal of the Triassic, with an estimated crown date at 166 Mya (127-205), during the Jurassic. Microthyriales split from Venturiales at 242 Mya (199-285), in the Triassic, with an estimated crown date at 181 Mya (133-230), in the Jurassic. The split between Zeloasperisporiales and Natipusillales is estimated at 195 Mya (148-243), in the early Jurassic. Their life styles are very different, Zeloasperisporiales are fungal epiphytes, while Natipusillales are freshwater fungi. The estimated crown date at 73 Mya (38-113) for Zeloasperisporiales and 69 Mya (34-111) for Natipusillales, is during the Cretaceous; however, they can be older once sufficient data is available. The MCC tree of Sordariomycetes provided by BEAST dates the split between Sordariomycetes and Leotiomycetes at 314 Mya (300-355), during the Carboniferous. The estimated date crowns at 283 Mya (235-331) for Sordariomycetes, in the Permian. The Meliolales crowns group shared the most common ancestor with Cephalothecaceae, Chaetosphaeriales, Coniochaetales, Cordanales, and Phyllachorales at 203 Mya (157-247), at the end of the Triassic. The Meliolales crown group is estimated at 135 Mya (93-177), during the Cretaceous. 


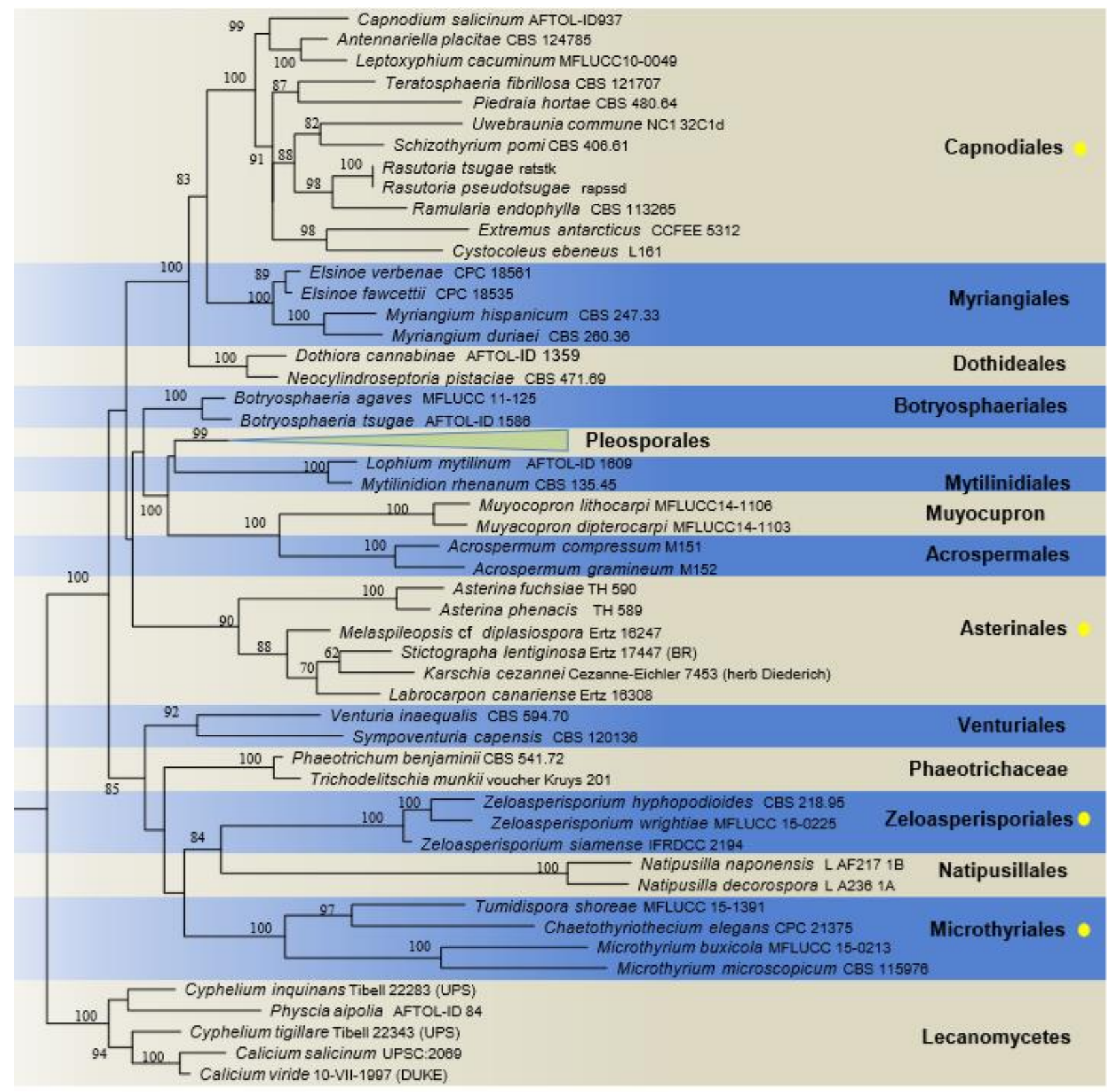

0.07

Fig. 5 - Phylogenetic tree for taxa of Dothideomycetes generated from maximum likelihood analysis of LSU, SSU, and RPB2 sequence data, including representative strains of fungal epiphytes. Bootstrap values above 50 are shown. Strain numbers are indicated after species names. The foliar epiphytes discussed in this study are marked with yellow dots.

\section{Discussion}

\section{Reconstruction of the evolutionary lineages}

The relaxed molecular clock allows more elastic modeling of rate heterogeneity, thus providing well-resolved phylogenetic results (Drummond et al. 2006). In this study, we have used only the fossils and calibration points estimated from previous studies that are reliable for our objectives. According to our target groups belonging to Dothideomycetes and Sordariomycetes, the divergence times of both classes were estimated by Pérez-Ortega et al. (2016). Thus, we used some calibration points from Pérez-Ortega et al. (2016) in our analysis, and also used the calibrations from fossil records in Dilcher (1965) and Beimforde et al. (2014). Although, there are 13 available fossil records for Ascomycota, we could not use them all in these analyses because each is suitable 




Fig. 6 - Phylogenetic tree of Sordariomycetes generated from maximum likelihood analysis of LSU, SSU, RPB1 and RPB2 sequence data, including representative strains of fungal epiphytes. Bootstrap values above 50 are shown. Strain numbers are indicated after species names. The foliar epiphytes discussed in this study are marked with yellow dot.

for focusing on individual groups of Ascomycota. They will however, provide potential calibrations when sufficient molecular data is available in the future (Beimforde et al. 2014). Therefore, we only used fossils that appeared to be identical to the modern specimens. Hence, we used fossils calibrations from Asterinales, Capnodiales and Microthyriales, and Calicium in Lecanoromycetes, plus the calibration points estimated for the Dothideomycetes crown group (Pérez-Ortega et al. 2016) in the maximum clade credibility (MCC) tree for Dothideomycetes. We used fossil calibrations for Meliolales, including the calibration points estimated for Sordariomycetes crown group and the split between Sordariomycetes and Leotiomycetes from Pérez-Ortega et al. (2016) in the MCC tree for Sordariomycetes. 
Table 2 Maximum likelihood analysis using internal calibrations from the fossils evidence and previous calibrations. Divergence times are listed in millions of years (Mya).

\begin{tabular}{lllll}
\hline & \multicolumn{1}{c}{$\begin{array}{c}\text { Time } \\
\text { (Mya) }\end{array}$} & \multicolumn{1}{c}{$\begin{array}{c}\text { Geological } \\
\text { period }\end{array}$} & $\begin{array}{c}\text { Pérez-Ortega et al. } \\
(\mathbf{2 0 1 6})\end{array}$ & $\begin{array}{c}\text { Beimforde et al. } \\
(\mathbf{2 0 1 4})\end{array}$ \\
\hline $\begin{array}{l}\text { Asterinales } \\
\text { crown group }\end{array}$ & $188(130-248)$ & Jurassic & - & - \\
\hline Capnodiales & $166(127-205)$ & Jurassic & - & - \\
\hline $\begin{array}{l}\text { Capnodiales }+ \\
\text { Myriangiales }\end{array}$ & $205(166-248)$ & Triassic-Jurassic & - & $350(273-459)$ \\
\hline $\begin{array}{l}\text { Dothideomycetes } \\
\text { crown group }\end{array}$ & $315(278-348)$ & Carboniferous & $290(241-349)$ & - \\
\hline $\begin{array}{l}\text { Meliolales } \\
\text { crown group }\end{array}$ & $135(93-177)$ & Cretaceous & - & - \\
\hline $\begin{array}{l}\text { Microthyriales } \\
\text { crown group }\end{array}$ & $181(133-230)$ & Jurassic & - & $260(207-339)$ \\
\hline $\begin{array}{l}\text { Sordariomycetes } \\
\text { crown group }\end{array}$ & $283(235-331)$ & Permian & $256(202-306)$ & $315(255-414)$ \\
\hline $\begin{array}{l}\text { Sordariomycetes }+ \\
\text { Leotiomycetes }\end{array}$ & $314(300-355)$ & Carboniferous & $290(242-353)$ & - \\
\hline $\begin{array}{l}\text { Zeloasperisporiales } \\
\text { crown group }\end{array}$ & $73(38-113)$ & Cretaceous & - & - \\
\hline $\begin{array}{l}\text { Zeloasperisporiales + } \\
\text { Natipusillales }\end{array}$ & $195(148-243)$ & Jurassic & - & \\
\hline
\end{tabular}

The phylogenetic tree with divergence estimation was topologically quite similar to the maximum likelihood phylogenetic tree in most of the major lineages within Dothideomycetes and Sordariomycetes (Figs. 5, 6). According to our target groups, topological differences were found in the clade of Asterinales and Microthyriales, but did not affect to the position of each species in other target groups. By using the maximum likelihood analysis (ML), Asterinales shared the most recent common ancestor (MRCA) with Botryosphaeriales, although such relationships were not clearly statistically supported, and are probably due to inadequate taxon sampling in the dataset (Fig. 7). The molecular clock tree provided by BEAST suggested that Asterinales is closely related and shared the most common ancestor with Dothideales, Myriangiales and Capnodiales in the Permian (Fig. 7), but it was unique, a supposition supported by moderate Bayesian posterior probability, based on available sequence data and fossil records. The speciation event in Asterinales and in Dothideales, Myriangiales and Capnodiales occurred in a different geological period. This is probably because numerous fungal taxa of Dothideomycetes have not yet been discovered and sequenced. More collections of species in Dothideomycetes are needed to resolve their evolutionary relationships. The Capnodiales split from Myriangiales at the end of the Triassic and the beginning of the Jurassic; however, the Myriangiales crown group is much younger than the Capnodiales crown group. The order Microthyriales is morphologically similar to Zeloasperisporiales in having thyriothecia and in addition, they are foliar epiphytes. On the other hand, Venturiales are saprobes or parasites on various plants with ascomata. The order Microthyriales shared the most common ancestor with Venturiales in the MCC tree, while sharing the most common ancestor with Natipusillales and Zeloasperisporiales in maximum likelihood analysis based on available fossil records and sequence data. The moderate support for both Asterinales and Microthyriales is mainly due to the insufficient taxon sampling. More collections are needed to fulfill the incomplete data. 


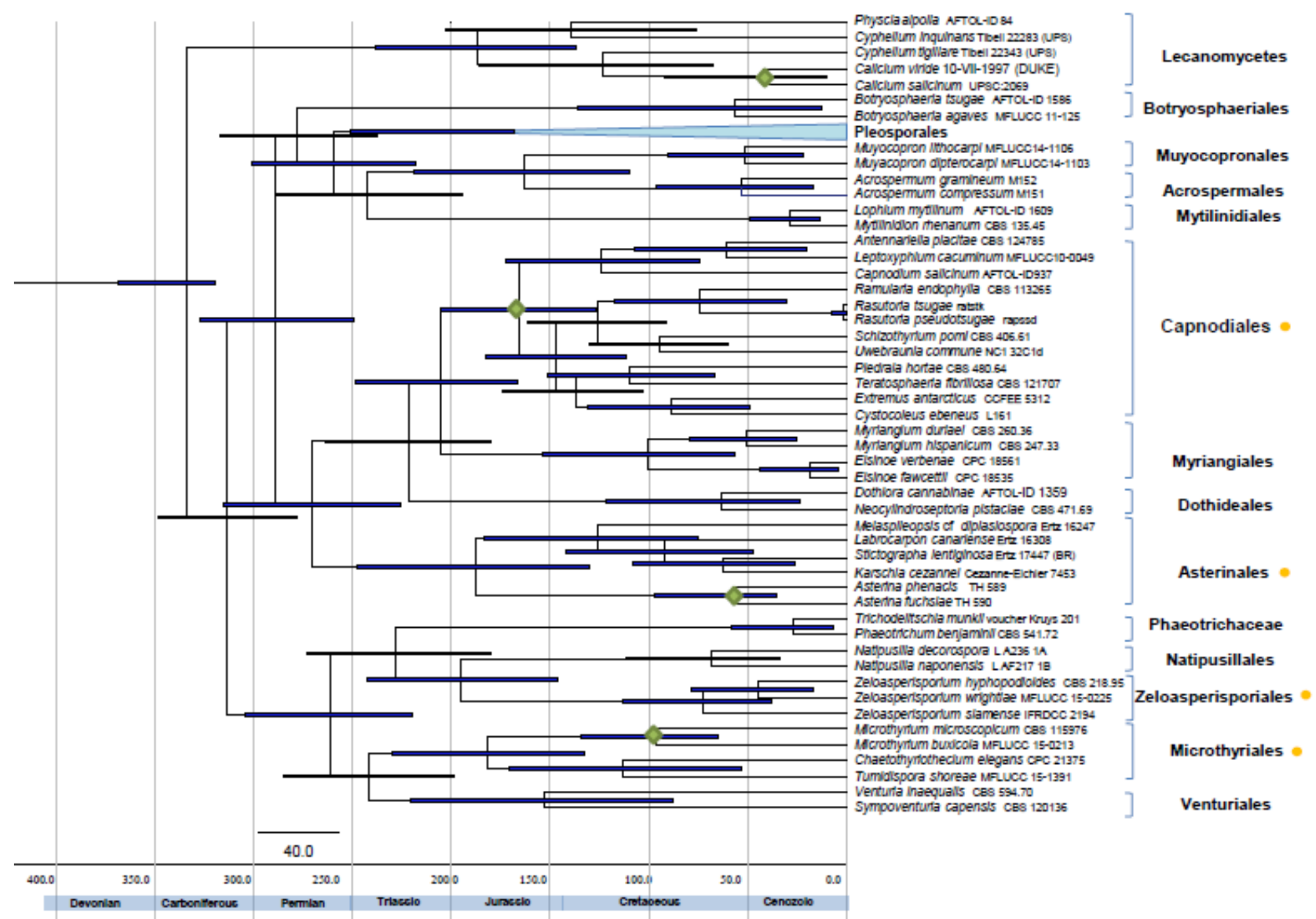

Fig. 7 - Divergence time estimations of Dothideomycetes tree obtained from a Bayesian approach (BEAST) using internal calibrations from fossil minimum age constraints and previous studies. Bars correspond to the 95\% highest posterior density (HPD) intervals. The fossil minimum age constraints and second calibrations used in this study are marked with green dots. Geological periods are indicated at the base of the tree. The foliar epiphytes discussed in this study are highlighted in purple. 


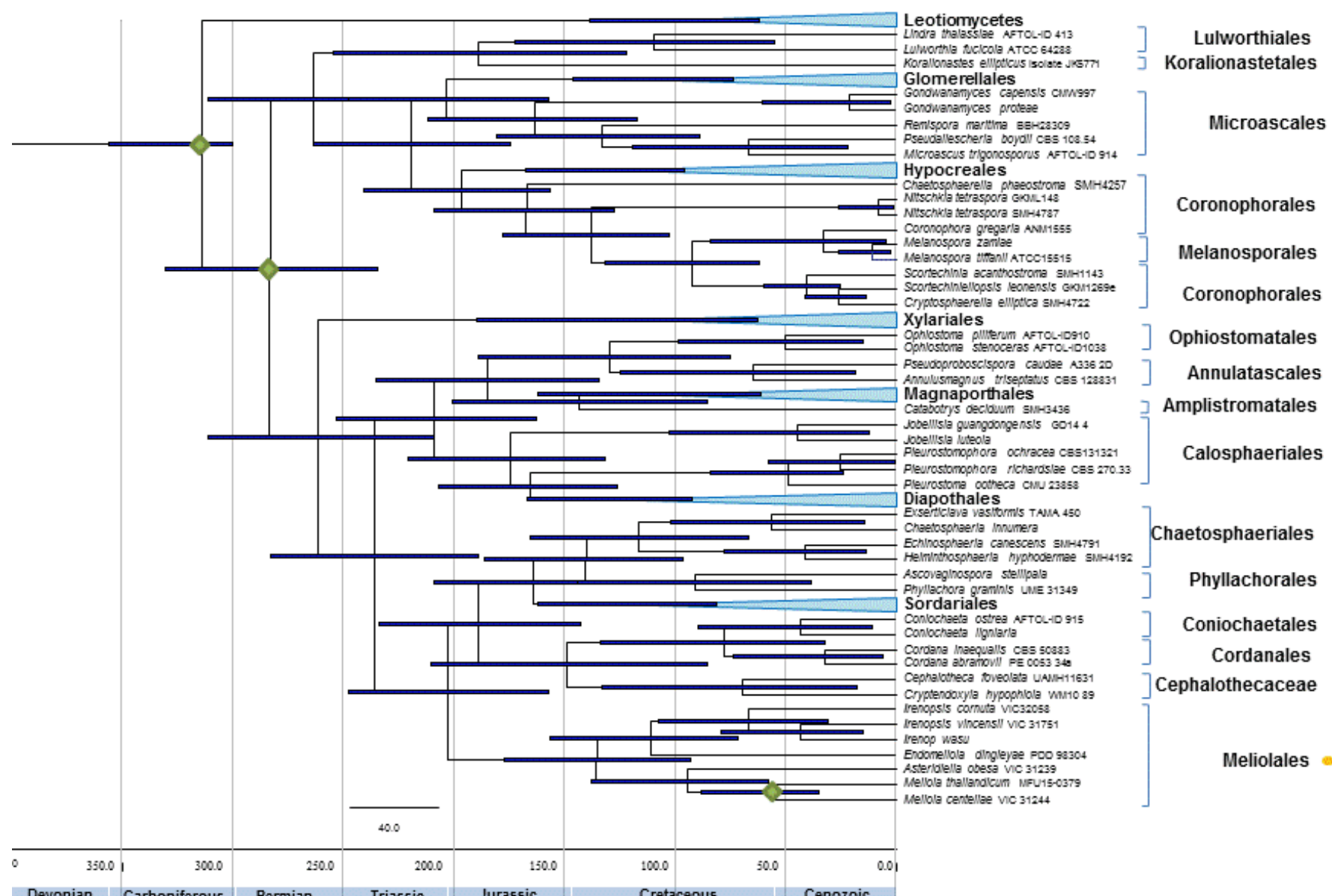

Fig. 8 - Divergence time estimations of Sordariomycetes tree obtained from a Bayesian approach (BEAST) using internal calibrations from fossil minimum age constraints and previous studies. Bars correspond to the 95\% highest posterior density (HPD) intervals. The fossil minimum age constraints and second calibrations used in this study are marked with green dots. Geological periods are indicated at the basal of the tree. The foliar epiphytes discussed in this study are highlighted in purple. 
The phylogenetic tree generated by maximum likelihood analysis of Sordariomycetes indicated that Meliolales is closely related to the clade comprising Cephalothecaceae, Coniochaetales and Cordanales, but is a distinct order, but the relationships are weakly supported. This result is similar to those of Hongsanan et al. (2015a) and Maharachchikumbura et al. (2015, 2016). The MCC tree for Sordariomycetes indicates that the Sordariomycetes crown group existed in the Permian. The Meliolales crown group is estimated to have evolved in the Cretaceous and its most common ancestors are Cephalothecaceae, Chaetosphaeriales, Coniochaetales, Cordanales, Phyllachorales, and Sordariales. However, these five orders clustered together, with Meliolales forming a distinct adjacent lineage. Accordingly, the Meliolales can be considered as relatively quite young, the crown group of this order is slightly distant from others.

\section{Divergence time estimates}

Divergence times estimated from our study used five calibration points for Dothideomycetes and three calibrations points for Sordariomycetes and generally correspond with the results of Beimforde et al. (2014). In the analysis of Dothideomycetes, our data included more calibration points within Dothideomycetes and Sordariomycetes. Furthermore, we did not use the external taxon in another phylum (e.g. Basidiomycetes), which may result to high ages in some lineages of Ascomycota. Compared with other studies, our results indicate neither much younger nor older when compared to Beimforde et al. (2014) and Pérez-Ortega et al. (2016).

Earlier studies used few representative strains from Dothideomycetes and Sordariomycetes to estimate the divergence times as aims of the studies were different. Thus, we are unable to compare individual lineages between our analysis and earlier studies. Divergence times estimated in this study correspond to Beimforde et al. (2014) and Pérez-Ortega et al. (2016) in the estimated date for the Dothideomycetes crown group. However, our analysis produced an older origin than Pérez-Ortega et al. (2016), while younger than Beimforde et al. (2014). The divergence time estimated for the Sordariomycetes crown group in our analysis was older than in Beimforde et al. (2014) and Pérez-Ortega et al. (2016), but younger than the estimated date of Gueidan et al. (2011). However, the Sordariomycetes crown group in the Permian in our analysis was the same as Beimforde et al. (2014) and Pérez-Ortega et al. (2016). The split between Leotiomycetes and Sordariomycetes estimated date in our analysis is most similar to Beimforde et al. (2014) (see Table 2), it is however older than the results from Prieto and Wedin (2013) and Pérez-Ortega et al. (2016). It is difficult to compare inferred ages estimated in each study due to various reasons such as genes under study, model of evolutionary rates, and parameter setting. The dating is based on fossil records and therefore depends on the period that a fossil specimen was discovered. In fact, the group may have evolved long before this period. Similarly, the dating resolved by the molecular clock is an indication of which taxa evolved on a chronological scale, but may be inaccurate due to insufficient data.

\section{Evolution of foliar epiphytes}

Foliar epiphytes in totally unrelated classes evolved at least in Permian (298.9 to 252.17 Mya) (Figs 7, 8). This is based on evidence from sequence data from representative foliar epiphytes and fossil calibrations. The estimated crown dates of most fungal epiphytes are in the Jurassic, with only Meliolales and Zeloasperisporiales in the Cretaceous. The evolution of the most closely related groups of fungi and foliar epiphytes occurred during the Triassic to Jurassic.

The order Asterinales is represented in this study by two strains of foliar epiphytes from Asterinaceae, which shared the most recent common ancestor with four lichenicolous strains of Melaspileaceae. This clade was synonymized under Asterotexiales based on the phylogenetic analysis of Ertz et al. (2016). Asterinales was represented as a clade unrelated to Asterotexiales in their study. Hyde et al. (2016) noted that both clades contain members of Asterinaceae and Parmulariaceae, thus they treated the older clade as Asterinales sensu stricto. Asterinaceae and Parmulariaceae were reported as polyphyletic by Inácio and Cannon (2008) and Guatimosim et al. (2015). The group of Asterinales sensu stricto was included in our analyses. 
The two families Asterinaceae and Melaspileaceae belong in Asterinales sensu stricto ( Asterotexiales) based on phylogenetic analysis (Ertz \& Diederich 2015). This is similar to our analyses (Fig. 5). Although Melaspileaceae species are lichenicolous, many Melaspileaceae strains lacking sequence data are saprobic or weakly lichenized (Ertz \& Diederich 2015). Ertz \& Diederich (2015) expect that many of the remaining Melaspileaceae species will be placed in Asterinales and presumably shared some characters of ascomata and life style (Ertz \& Diederich 2015).

The orders Asterinales, Capnodiales, Dothideales and Myriangiales have the same common ancestor, with possible origins in the Permian. The uniqueness of superficial hyphae with appressoria, and thyriothecia with "star"-like openings are typical of foliar epiphytes in Asterinales. The lichenicolous (Melaspileaceae) in Asterinales also share with Asterinaceae, some characters such as clavate with 8-spored asci and 1-septate, brown ascospores. Some species in Capnodiales and Myriangiales have similar flattened ascomata, as Asterinaceae species, but lack "star"-like openings and superficial hyphae with appressoria. However, they are unrelated to the Asterinales clade. The divergence time estimates for Asterinales are approximately in the Jurassic, while the split nodes of Dothideales, Capnodiales, and Myriangiales are approximately at the middle of Triassic, which suggest Asterinales evolved later.

The origin of land plants are major structural components of terrestrial ecosystems which led to important changes in the environment (Kenrick \& Crane 1997, Lewis \& McCourt 2004; O'Kelly 2007), and is dated approximately at 476-432 Mya (McCourt et al. 2004, Leliaert et al. 2011). Because the crown node estimated for Asterina appears in the Cretaceous to Cenozoic, thus we presume that foliar epiphytes in Asterinales may already have been associated with plants at least in the Cretaceous. No fossil for Asterinales has so far been discovered in pre-Cretaceous.

In our study, Capnodiales comprise three strains of Capnodiaceae, which are closely related to the clade containing Dissoconiaceae, Euantennariaceae, Extremaceae, Mycospherellaceae, Schizothyriaceae, and Teratosphaeriaceae. The family Capnodiaceae has unique morphological characters. Foliar epiphytes in Capnodiaceae feed on honey dew excreted by insects (Chomnunti et al. 2011, 2014). Others families within Capnodiales presumably evolved from Capnodiaceae (Fig. 7). Some groups in Capnodiales have the ability to reproduce and survive in specific habitats, such as the rock-inhabiting fungi (i.e. Extremus antarcticus and Cystocoleus ebeneus) occur at the base of Capnodiales. Over time, species might have diverged and adapted to changing environments several times.

Species of Capnodiales mostly have superficial ascomata, but they are immersed in some species of Mycosphaerellaceae and Teratosphaeriaceae (represented by Ramularia endophylla and Teratosphaeria fibrillosa in our analysis, Fig. 7). Schizothyrium pomi (Schizothyriaceae) and Uwebraunia commune (Dissoconiaceae) are flyspeck and sooty blotch fungi, with have completely superficial ascomata, appearing as small black dots, on the cuticle of plants. The superficial hyphae of foliar epiphytes from these families can coat the surface of plants. Piedraia hortae (Piedraiaceae) is a pathogen in humans causing 'black piedra' in hair. It is therefore important to understand how this species evolved and became pathogenic, but we are unable to establish the evolutionary relationships of Piedraiaceae with our dataset herein.

Sooty moulds in Capnodiaceae live on plant surfaces and feed on the honeydew from insects (Hughes 1976, Faull et al. 2002, Auclair 1963). The first aphid fossil was dated to the middle of Triassic (Szwedo \& Nel 2011), and sucking insects with sucking beaks are known from the Carboniferous (Labandeira 2006, Nel et al. 2013). Thus, associations between sooty moulds and honeydew-producing insects may have evolved before the Cretaceous (Schmidt et al. 2014). Foliar epiphytes in Capnodiales were also presumably associated with plants at least in the early Cretaceous based on the oldest fossil evidence from Metacapnodiaceae. This family provides the fossil calibration for the crown node of Capnodiales with an estimated date in the Cretaceous (Fig. 7). No fossil evidence for Capnodiales has been found before the Cretaceous. Based on evidence from the earliest diverging lineages, Phaeotheca and Comminutispora (Crous et al. 2007), the ancestral nutritional mode of ancestors of Capnodiales are likely to have been saprobic. However, Ismail et al. (2016) indicated that the ancestral nutritional mode of Capnodiales ancestor is likely to 
have been plant parasites. The different families of Capnodiales appear to have evolved several times, with different lifestyles (e.g. Capnodium salicinum as saprobes and Piedraia hortae as human pathogens), however, the taxa in the later diverging clades tend to be strictly necrotrophic plant pathogens (Crous et al. 2007). Capnodiales will probably comprise numerous divergent groups with different lifestyles, once adequate sequence data from a wider number of species are analysed.

The Meliolales crown group diverged in the Cretaceous (Fig. 8). Species in this order are mostly biotrophic parasites, as they have superficial hyphae with hyphopodia. They form a distinct lineage at the base of Sordariomycetes. Thus, they were represented as the subclass Meliolomycetidae (Maharachchikumbura et al. 2015, 2016). The ancestral nutritional mode of Meliolales is likely to have been saprobic or weakly parasitic; this presumption is based on the relationships between Meliolales and other ancestral saprobic orders of Sordariomycetes. The lineage of Meliolales has therefore evolved to be specific plant pathogens and are not saprobes. Within the Meliolales, species have evolved the unique characters of superficial hyphae with hyphopodia, which obtain nutrients from living plants (Hongsanan et al. 2015a). The genus Meliola and Asteridella obesa evolved from a common ancestor and the former have reduced outer, conical, peridial cells and produce setae on superficial hyphae. The associations between Meliolales and plants presumably evolved at least in the Cretaceous, based on the divergence estimates, although fossil specimens of Meliolales have only been found in the Eocene. This coincides with the major periods of radiation and spread of Angiosperms (Slippers et al. 2013).

In this study, the order Microthyriales form a sister group with Venturiales and share a common ancestor, but this relationship is not well-resolved (Fig. 7). The earlier ancestral node comprises Microthyriales, Natipusillales, Phaeotrichaceae and Zeloasperisporiales. Microthyriales and Zeloasperisporiales are foliar epiphytes, while Natipusillales and Phaeotrichaceae are freshwater fungi and dung fungi, respectively (Fig. 7). The appearance of freshwater and dung fungi in this node demonstrates that different lifestyles have evolved several times (Vijaykrishna et al. 2006). Since Zeloasperisporiales and Microthyriales are a recent lineage, we conclude that foliar epiphytes within this ancestral node have evolved the thyriothecia, later than other foliar epiphytes. As little sequence data is available for Microthyriales, thus we do not discuss the evolution of genus/species here.

\section{Acknowledgements}

Kevin D. Hyde thanks the Chinese Academy of Sciences, project number 2013T2S0030, for the award of Visiting Professorship for Senior International Scientists at Kunming Institute of Botany.

\section{References}

Ariyawansa HA, Hyde KD, Jayasiri SC, Buyck B et al. 2015 - Fungal Diversity Notes 111-246 Taxonomic and phylogenetic contributions to fungal taxa. Fungal Diversity 75, 27-274.

Arnaud G 1918 - Lés Asterinées. Annals d'École National d'Agriculture de Montpellier Série 2 16, $1-288$.

Auclair JL. 1963 - Aphid feeding and nutrition. Annual Review Entomology 8, 439-490.

Barr ME. 1987 - New taxa and combinations in the Louculoascomycetes. Mycotaxon 29, 501-505.

Beimforde C, Feldbergb K, Nylinderc S, Rikkinend J et al. 2014 - Estimating the Phanerozoic history of the Ascomycota lineages: Combining fossil and molecular data. Molecular Phylogenetics and Evolution 78, 386-398.

Benton MJ, Donoghue PCJ, Asher RJ. 2009 - Calibrating and constraining molecular clocks S.B. Hedges, S. Kumar (Eds.), Timetree of Life, University Press, Oxford: 35-86.

Berbee ML, Taylor JW. 1993 - Dating the evolutionary radiations of the true Fungi. Canadian Journal of Botany 71, 1114-1127. 
Berbee ML, Taylor TW. 2007 - Rhynie chert: a window into a lost world of complex plant-fungus interactions. New Phytologist 174, 475-479.

Berbee ML, Taylor JW. 2010 - Dating the molecular clock in Fungi - how close are we? Fungal Biology Reviews 24, 1-15.

Cai L, Jeewon R, Hyde KD. 2006 - Phylogenetic investigations of Sordariaceae based on multiple gene sequences and morphology. Mycological Research 110, 137-150.

Cai L, Guo XY, Hyde KD. 2008 -. Morphological and molecular characterization of a new anamorphic genus Cheirosporium, from freshwater in China. Persoonia 20: 53-58.

Castañeda RF, Fabre DE, Parra M, Perez M, Guarro J. 1996 - Some airborne conidial fungi from Cuba. Mycotaxon 60, 283-290.

Chomnunti P, Schoch CL, Aguirre-Hudson B, Ko-Ko TW et al. 2011 - Capnodiaceae. Fungal Diversity 51, 103-134.

Chomnunti P, Ko Ko TW, Chukeatirote E, Hyde KD et al. 2012 - Phylogeny of Chaetothyriaceae in northern Thailand including three new species. Mycologia 104, 382-395.

Chomnunti P, Hongsanan S, Hudson BA, Tian Q et al. 2014 - The sooty moulds. Fungal Diversity $66,1-36$.

Cooksoon IC. 1947 - Fossil fungi from Tertiary deposits in the southern hemisphere: Part I. Proceedings of the Linnean Society of New South Wales 72, 207-214.

Crous PW, Schubert K, Braun U, Hoog De GS et al. 2007 - Opportunistic, human-pathogenic species in the Herpotrichiellaceae are phenotypically similar to saprobic or phytopathogenic species in the Venturiaceae. Studies in Mycology 58, 185-217.

Dilcher DL. 1965 - Epiphyllous fungi from Eocene deposits in western Tentnessee, U.S.A. Palaeontographica, Beiträge zur Naturgeschichte der Vorzeit 116, 1-54.

Doi Y, Uemura K. 1985 - Fossil Microthyrium on Buxus leaf compressions from the Upper Miocene, and its living relative in Japan. Bulletin of the National Science Museum. Series B (Botany) 11, 127-136.

Drummond AJ, Ho SYW, Phillips MJ, Rambaut A. 2006 - Relaxed phylogenetics and dating with confidence. PLoS Biology 4, 699-710.

Engelhardt H, Kinkelin F. 1908 - Obcrphocäne Flora und Fauna des Untermaiutales. insbesondere des Frankfurter Klärbeckens. - Abhandlungen der Senckcnberg. Naturforsch Gesellschaft 29, 150-306.

Eriksson B. 1978 - Fossi microthyriaceous fungi from Tervola, northern Finland. Annales Botanici Fennici 15, 122-127.

Ertz D, Diederich P. 2015 - Dismantling Melaspileaceae: a first phylogenetic study of Buelliella, Hemigrapha, Karschia, Labrocarpon and Melaspilea. Fungal Diversity 71:141-164.

Ertz D, Heuchert B, Braun U, Freebury CE et al. 2016 - Contribution to the phylogeny and taxonomy of the genus Taeniolella, with a focus on lichenicolous taxa. Fungal Biology 120, $1416-1447$.

Faull JL, Olejnik I, Ingrouille M, Reynolds D. 2002 - A reassessment of the taxonomy of some tropical sooty moulds. Tropical Mycology 2, 33-40.

Forest F. 2009 - Calibrating the Tree of Life: fossils, molecules and evolutionary timescales. Annals of Botany 104, 789-794.

Frantz U. 1959 - Die Pollenflora der Braunkohle von Lohsa/Nied erlausitz. - JnauguralDissertation zur Erlangung der Doktorwürde der Math.-Naiurv Fakultäl der Freien Univ. Berlin. 46 p.

Germeraad JH. 1979 - Fossil remains of fungi, algae and other organisms from Jamaica. Cripta Geologica 52, 1-41.

Gilbert G, Reynolds DR. 2002 - The ecology of foliicolous fungi. In 'Proceedings of the $7^{\text {th }}$ international mycological congress' (Ed. L Ryvarden) p. 89. (Oslo)

Gilbert G, Reynolds DR. 2005 - Epifoliar fungi from Queensland, Australia. Australian Systematic Botany 18, 265-289. 
Givulescu R. 1971 - Zwei Microthyriaceen aus dem neogen Rumäniens. Zeitschrift für Pilzkunde 37, 1-4.

Godwin H, Andrew R. 1951 - A fungal fruit body common in post-glacial peat deposits. New Phvtologist 50, 179-183.

Guatimosim E, Firmino AL, Bezerra JZ, Pereira OL et al. 2015 - Towards a phylogenetic reappraisal of Parmulariaceae and Asterinaceae (Dothideomycetes). Persoonia 35, 230-241.

Gueidan C, Ruibal C, de Hoog GS, Schneider H. 2011 - Rock-inhabiting fungi originated during periods of dry climate in the late Devonian and middle Triassic. Fungal Biology 115, 987996.

Hall TA. 1999 - BioEdit: a user-friendly biological sequence alignment editor and analysis program for Windows 95/98/NT. Nucleic Acids Symposium Series 41, 95-98.

Hansford CG. 1961 - The Meliolaceae a monograph. Sydowia Beiheft 2, 1-806

Heckman DS, Geiser DM, Eidell BR, Stauffer RL et al. 2001 - Molecular evidence for the early colonization of land by fungi and plants. Science 293, 1120-1133.

Hedman MH 2010 - Constraints on clade ages from fossil outgroups Paleobiology 36, 16-31.

Hongsanan S, Li YM, Liu JK, Hofmann T et al. 2014a - Revision of genera in Asterinales. Fungal Diversity 68, 1-68.

Hongsanan S, Chomnunti P, Crous PW, Chukeatirote E, Hyde KD. 2014b - Introducing Chaetothyriothecium, a new genus of Microthyriales. Phytotaxa 161, 157-164.

Hongsanan S, Tian Q, Peršoh D, Zeng XY et al. 2015a - Meliolales. Fungal Diversity 74, 1-51.

Hongsanan S, Tian Q, Bahkali AH, Yang JB et al. 2015b - Zeloasperisporiales ord. nov., and two new species of Zeloasperisporium. Cryptogamie Mycologie 36, 301-317.

Hongsanan S, Hyde KD, Bahkali AH, Camporesi E et al. 2015c - Fungal Biodiversity Profiles 11-20. Cryptogamie Mycologie 36, 355-380.

Huelsenbeck, J.P. \& Ronquist, F. 2001 - MRBAYES: Bayesian inference of phylogenetic trees. Bioinformatics 17, 754-755.

Hughes SJ. 1976 - Sooty moulds. Mycologia 68, 693-820.

Hughes SJ, Seifert KA. 2012 - Taxonomic and nomenclatural notes on sooty mould name based on species mixtures: Hormiscium handelii and Torula lecheriana. Mycoscience 53, 17-24.

Hyde KD, Jones EBG, Liu JK, Ariyawansa H et al. 2013 - Families of Dothideomycetes. Fungal Diversity 63, 1-313.

Hyde KD, Hongsanan S, Jeewon R, Bhat DJ et al. 2016 - Fungal diversity notes 367-490: taxonomic and phylogenetic contributions to fungal taxa. Fungal Diversity 80, 1-270.

Inácio CA, Cannon PF 2008 - The genera of the Parmulariaceae. CBS Fungal Biodiversity Series $8,1-195$.

Inoue J, Donoghue PCJ, Yang Z. 2010 - The impact of the representation of fossil calibrations on Bayesian estimation of species divergence times. Systematic Biology 59, 74-89.

Ismail SI, Batzer JC, Harrington TC, Crous PW et al. 2016 - Ancestral state reconstruction infers phytopathogenic origins of sooty blotch and flyspeck fungi on apple. Mycologia 108, $15-36$.

Justavino DR, Kirschner R, Piepenbring M. 2015 - New species and new records of Meliolaceae from Panama. Fungal Diversity 70, 73-84.

Katoh K, Asimenos G, Toh H. 2009 - Multiple alignment of DNA sequences with MAFFT. Methods in Molecular Biology 537, 39-64.

Kenrick P, Crane PR. 1997 - The origin and early diversification of land plants: a cladistic study, p. 441. Washington, DC: Smithsonian Institution Press.

Kirk PM, Cannon PF, David JC, Stalpers JA. 2001 - Ainsworth\& Bisby's Dictionary of the fungi, 9th edn. CABI, Wallingford

Kirk PM, Cannon, PF, Minter DW, Stalpers JA. 2008 - Ainsworth \& Bisby's dictionary of the fungi, $10^{\text {th }}$ edition. CAB International, Wallingford, UK, $428 \mathrm{p}$. 
Köck C. 1939 - Fossile Kryptogamen aus del eozaneu Braunkohle des Gciseltalcs. Nova Acta Academiae Caesareae Leopoldino-Carolinae Germanicae Naturae Curiosorum. Verhandlungen der Kaiserlich 6, 333-359.

Labandeira CC. 2006 - The four phases of plant-arthropod associations in deep time. Geologica Acta 4, 409-438.

Lancucka-Srodoniowa M. 1958 - Salviiila and Azolla in the Miocene of Poland. Acta Biologica Cracoviensia 1, 15-23.

Leliaert F, Verbruggen H, Zechman FW. 2011 - Into the deep: new discoveries at the base of the green plant phylogeny. BioEssays 33, 683-669.

Lewis LA, McCourt RM. 2004 - Green algae and the origin of land plants. American Journal of Botany $91,1535-1556$.

Li GJ, Hyde KD, Zhao RN, Hongsanan S et al. 2016 - Fungal diversity notes 253-366: taxonomic and phylogenetic contributions to fungal taxa. Fungal Diversity 78, 1-237.

Lukoschek V, Keogh JC, Avise JC. 2012 - Evaluating fossil calibrations for dating phylogenies in light of rates of molecular evolution: a comparison of three approaches. Systematic Biology 61, 22-43.

Luttrell ES. 1973 - Loculoascomycetes. In: Ainsworth, G.C., Sparrow, F.K. \& Sussman, A.S. (eds) The fungi. An advanced treatise. Academic Press, New York and London, pp. 135-219.

Magallon SA 2010 - Using fossils to break long branches in molecular dating: a comparison of relaxed clocks allied to the origin of angiosperms. Systematic Biology 59, 384-399.

Maharachchikumbura SSN, Hyde KD, Jones EBG, McKenzie EHC et al. 2015 - Towards a natural classification and backbone tree for Sordariomycetes. Fungal Diversity 72, 199-301.

Maharachchikumbura SSN, Hyde KD, Jones EBJ, McKenzie EHC et al. 2016 - Families of Sordariomycetes. Fungal Diversity 79, 1-317.

Marshall CR. 2008 - A simple method for bracketing absolute divergence times on molecular phylogenies using multiple fossil calibration points. The American Naturalist 171, 726-742.

McCourt RM, Delwiche CF, Karol KG. 2004 - Charophyte algae and land plant origins. Trends in Ecology and Evolution 19, 661-666.

Nel A, Prokop J, Nel P, Grandcolas P et al. 2013 - Environmental health and safety considerations for nanotechnology. Chemical Research 46, 605-606.

Néraudeau D, Perrichot V, Dejax J, Masure E et al. 2002 - Un nouveau gisement à ambre insectifère et à végétaux (Albien terminal probable: Archingeay (Charente-Maritime, France). Geobios 35, 233-240.

O'Kelly CJ. 2007 - The origin and early evolution of green plants. In: Falkowski PG, Knoll AH (eds) Evolution of Primary Producers in the Sea. Elsevier Academic, Burlington, pp. 287309.

Parham JF, Donoghue PCJ, Bell CJ, Calway TD, Head JJ, et al. 2012 - Best practices for justifying fossil calibrations. Systematic Biology 61, 346-359.

Pérez-Ortega S, Garrido-Benavent I, Grube M, Olmo R, Ríos A. 2016 - Hidden diversity of marine borderline lichens and a new order of fungi: Collemopsidiales (Dothideomyceta). Fungal Diversity. doi:10.1007/s13225-016-0361-1

Perrichot V, Néraudeau D, Tafforeau P. 2010 - Charentese amber. In: Penney, D. (ed.). Biodiversity of fossils in amber from the major world deposits. Manchester, Siri Scientific Press, 192-207.

Prieto M, Wedin M. 2013 - Dating the diversification of the major lineages of Ascomycota (Fungi). PLOS One 8, e65576

Pyron RA. 2010 - A likelihood method for assessing molecular divergence time estimates and the placement of fossil calibrations. Systematic Biology 59, 185-195.

Rambaut A. 2006 - FigTree. Tree figure drawing tool version 1.3.1, Institute of Evolutionary Biology, University of Edinburgh. <http://tree.bio.ed.ac.uk/software/figtree/>.

Reynolds DR. 1998 - Capnodiaceous sooty mold phylogeny. Canadian Journal of Botany 76, 2125-2130. 
Roumeguère C. 1880 - Fungi Gallici exsiccati, cent. X. Revue Mycologique Toulouse. 2, 200-202.

Ruibal C, Gueidan C, Selbmann L, de Hoog S. 2009 - Phylogeny of rock-inhabiting fungi related to Dothideomycetes. Studies in Mycology 64, 123-133.

Sanderson MJ. 2003 - Molecular data from 27 proteins do not support a Precambrian origin of land plants. American Journal of Botany 90, 954-956.

Sauquet H, Ho SY, Gandolfo MA, Jordan GJ et al. et al. 2010 - Testing the impact of calibration on molecular divergence times using a fossil-rich group: the case of Nothofagus, Fagales). Systematic Biology 61, 289-313.

Schmidt AR, Beimforde C, Seyfullah LJ, Wege SE et al. 2014 - Amber fossils of sooty moulds. Review of Palaeobotany and Palynology 200, 53-64.

Schoch CL, Crous PW, Groenewald JZ, Boehm EW et al. 2009 - A class-wide phylogenetic assessment of Dothideomycetes. Studies in Mycology 64, 1-15.

Selbmann L, de Hoog GS, Zucconi L, Isola D, Onofri S. 2014 - Black yeasts in cold habitats. In: Margesin R, Buzzini P (eds) Cold-adapted yeasts. Springer, Berlin, Heidelberg, pp 173189.

Selkirk DR. 1975 - Tertiary fossil fungi from Kiandra, New South Wales. Proceedings of the Linnean Society of New South Wales 100, 70-94.

Simon L, Bousquet J, Levesque RC, Lalonde M. 1993 - Origin and diversification of endomycorrhizal fungi and coincidence with vascular land plants. Nature 363, 67-69.

Slippers B, Boissin E, Phillips AJL, Groenewald JZ et al. 2013 - Phylogenetic lineages in the Botryosphaeriales: a systematic and evolutionary framework. Studies in Mycology 76, 3149.

Szafer W. 1961 - Miocenska flora ze starych gliwic na slasku. Instytut Geologiezny Prace 33, 1205.

Szwedo J, Nel A. 2011 - The oldest aphid insect from the Middle Triassic of the Vosges, France. Acta Palaeontologica Polonica 56, 757-766.

Taylor JW, Berbee ML. 2006 - Dating divergences in the Fungal Tree of Life: review and new analyses. Mycologia 98, 838-849.

Thomas J, Alex TE, Thomas RJ. 2013 - Meliola marthomaensis sp. nov. an addition to Meliolaceae from Western Ghat Region in Kerala State, India. Universal Journal of Plant Science 1, 100-103.

Vijaykrishna D, Jeewon R, Hyde KD 2006 - Molecular taxonomy, origins and evolution of freshwater ascomycetes. Fungal Diversity 23, 351-390.

Von Arx JA von, Müller E. 1975 - A re-evaluation of the bitunicate ascomycetes with key to families and genera. Studies in Mycology 9, 1-159.

Wilkinson RD, Steiper ME, Soligo C, Martin RD et al. 2011 - Dating primate divergences through and integrated analysis of paleontological and molecular data. Systematic Biology 60, 1631.

Winka K, Eriksson O, Bång A. 1998 - Molecular evidence for recognizing the Chaetothyriales. Mycologia 90, 822-830.

Wu HX, Schoch CL, Boonmee S, Bahkali AH et al. 2011 - A reappraisal of Microthyriaceae. Fungal Diversity 51, 189-248.

Zhao RL, Zhou JL, Chen J, Margaritescu S et al. 2016 - Towards standardizing taxonomic ranks using divergence times - a case study for reconstruction of the Agaricus taxonomic system. Fungal Diversity 78, 239-292.

Zhaxybayeva O, Gogarten JP. 2002 - Bootstrap, Bayesian probability and maximum likelihood mapping: exploring new tools for comparative genome analyses. BMC Genomics 3, 4. 